\title{
Effect of evaporation through nanoporous medium on diffusiophoresis
}

\author{
Dokeun Lee ${ }^{1}$, Junsuk Kim ${ }^{1}$, Hyomin Lee ${ }^{2^{*}}$ and Sung Jae Kim ${ }^{1,3,4^{*}}$ (D)
}

\begin{abstract}
Diffusiophoresis near a nanoporous medium is the phenomenon that particles were spontaneously moved from low concentration region to high concentration region. However, unidirectional particle motion impeded further application of this phenomenon and lack of studies about the external convective flow effect such as evaporation through a nanoporous medium were reported. In this work, we investigated the evaporation effect through a nanoporous medium on spontaneous particle exclusion zone induced by diffusiophoresis. Consequently, particle's motion was divided into three regimes: diffusiophoresis regime, transition regime, and evaporation regime depending on the evaporation effect. The experiment in which the time of initiating evaporation effect was controlled by adjusting the region of PDMS on a nanoporous medium showed that evaporation plays a critical role in studying the spontaneous particle exclusion zone. This rigorous analysis would provide a useful strategy for optimizing a spontaneous particle preconcentration/detection platform.
\end{abstract}

Keywords: Diffusiophoresis, Nanoporous medium, Evaporation, Spontaneous particle exclusion zone

\section{Introduction}

Diffusiophoresis is the electrochemical phenomenon that a charged particle moved from low concentration region to high concentration region along a concentration gradient in the case of electrolyte solution such as $\mathrm{KCl}$ solution where each diffusivity is almost identical so that one can ignore induced electrophoresis. This concentration gradient can be generated by two miscible liquids of different concentrations $[1,2]$ or by ion exchange [3-5]. Especially, cation exchange phenomenon can occur near a nanoporous medium having protons with high diffusivity if the medium meets the solution with non-protonic cation, which means that the concentration gradient would be spontaneously generated by cations' difference of diffusivity without any external forces [4]. Therefore, this spontaneously induced concentration gradient forces

\footnotetext{
*Correspondence: fluid@jejunu.ac.kr; gates@snu.ac.kr

1 Department of Electrical and Computer Engineering, Seoul National University, Seoul 08826, Republic of Korea

${ }^{2}$ Department of Chemical and Biological Engineering, Jeju National

University, Jeju 63243, Republic of Korea

Full list of author information is available at the end of the article
}

a charged particle to move away from the medium so that the medium should be particle-free to form a spontaneous particle exclusion zone. This mechanism has been utilized for desalting/purification applications [5-7].

However, there is lack of studies about the effect of the external convective flow through a nanoporous medium such as evaporation on the spontaneous motion of a particle in dead-end microchannel. A convective flow due to the imbibition through nanoporous medium has been found to play non-negligible role for diffusiophoresis of charged particles [3], while this imbibition has been conventionally neglected. Therefore, in this work, we would investigate the relationship between another kind of convective flow due to evaporation when a nanoporous medium was exposed to air and the spontaneous particle exclusion zone near the medium. Figure 1 showed the schematic of a micro/nanofluidic device having the nanoporous medium which is partially covered with PDMS piece (i.e. open to ambient). Also, the particle inside the microchannel is affected by diffusiophoretic velocity, imbibition velocity, and evaporation velocity as 


\section{a}

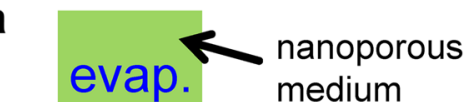

evap. medium

nir

negatively charged microparticle

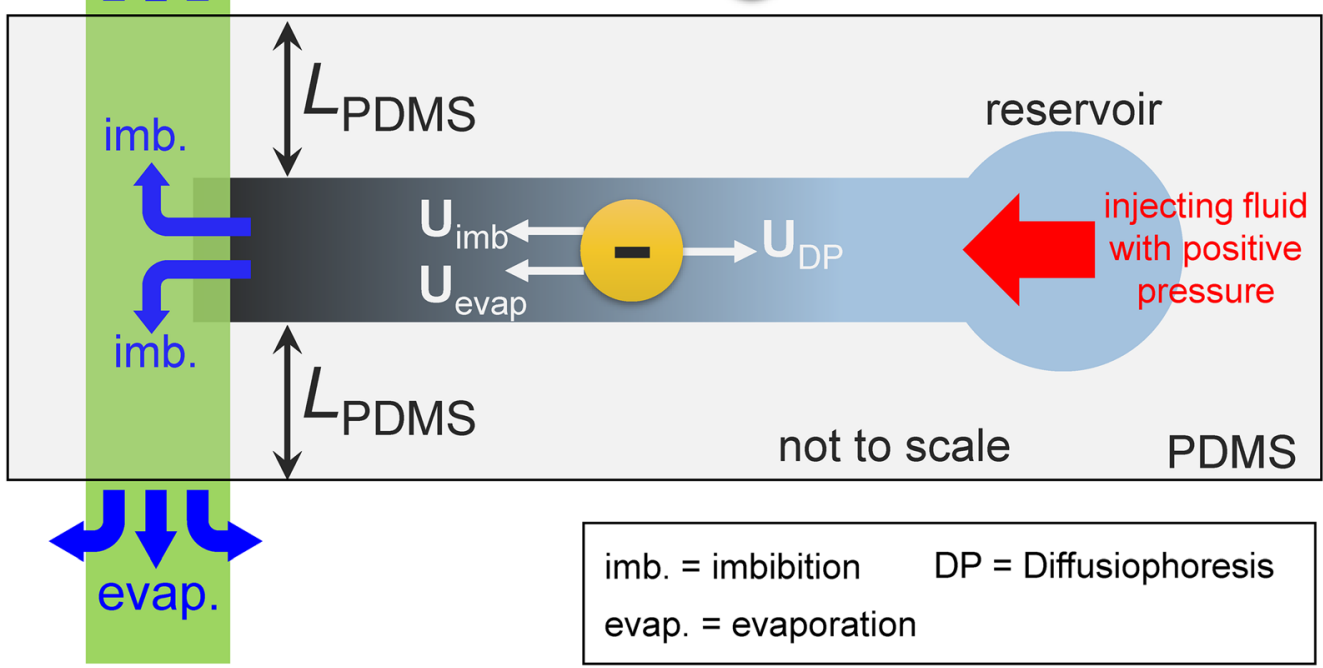

b

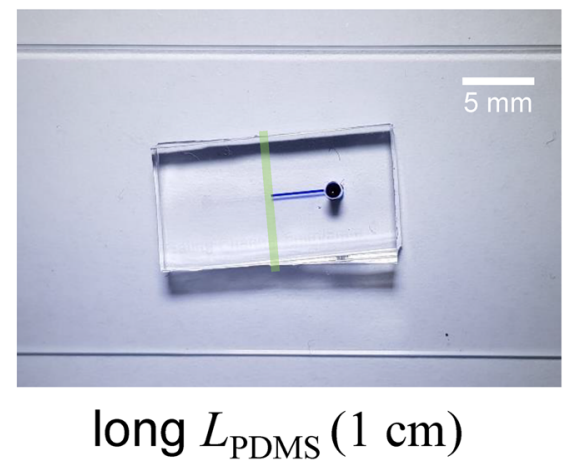

C

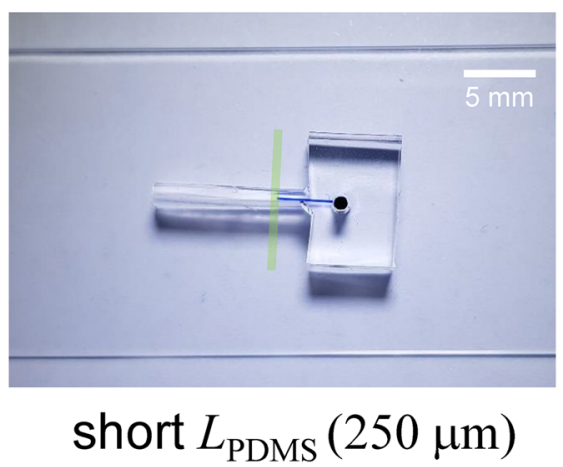

Fig. 1 a The schematic of spontaneous particle exclusion zone induced by diffusiophoreis near the nanoporous medium. $U_{\text {imb }}$ is the imbibition velocity, $U_{D P}$ is the diffusiophoretic velocity, $U_{\text {evap }}$ is the velocity induced by evaporation through the medium. $L_{\text {PDMS }}$ is the distance of PDMS between the channel and the medium exposed to air (the location of starting evaporation). Fabricated devices of $L_{\text {PDMS: }}: \mathbf{b} 1 \mathrm{~cm}$ and $\mathbf{c} 250 \mu \mathrm{m}$

depicted. Diffusiophoretic velocity's direction is the opposite of imbibition velocity and evaporation velocity. The effect of evaporation through a nanoporous medium can be adjusted by the distance of PDMS piece between the microchannel and the medium exposed to air $\left(L_{\mathrm{PDMS}}\right)$. As a result, the particle motion was largely affected by the evaporation as well as the imbibition through nanoporous medium, suggesting a fundamental basis for designing spontaneous particle separation or preconcentration platform.

\section{Materials and methods}

Device fabrication

The micro/nanofluidic device used in this study was fabricated using polydimethyl-siloxane (PDMS, Sylgard 184 silicone elastomer kit, DowCorning, USA) incorporated with Nafion nanoporous membrane. The PDMS building blocks of the devices were fabricated by following general soft-lithographical method [8]. The mold patterning microchannels was fabricated through silicon wafer using photo lithography. The base and curing agents of 
polydimethyl-siloxane (PDMS) were mixed in a ratio of 10:1 and degassed in a vacuum chamber for $1 \mathrm{~h}$. The mixed solution was poured onto a mold wafer and cured in an oven for $4 \mathrm{~h}$ at $75^{\circ} \mathrm{C}$.

A straight microchannel was orthogonally aligned with Nafion patterning as shown in Fig. 1a. The microchannel had a dimension of $15 \mu \mathrm{m}$ thickness, $200 \mu \mathrm{m}$ width and $0.5 \mathrm{~cm}$ length. Instead of sophisticated nano-lithographical fabrication, a surface patterning method $[9,10]$ of Nafion solution (20 wt\% resin solution, Sigma Aldrich) was employed to connected microchannels. Briefly, Nafion solution was injected inside a straight PDMS microchannel and the microchannel was detached. Remaining Nafion liquid was dried for $10 \mathrm{~min}$ at $95{ }^{\circ} \mathrm{C}$. Then, pre-fabricated PDMS piece which had microchannel networks was aligned under microscopic observation and bonded with plasma bonder (CuteMP, Femto Science, Korea) as shown in Fig. 1a. Assembled devices of long $L_{P D M S}$ and short $L_{P D M S}$ were shown in Fig. 1b, c, respectively.

\section{Chemical preparation}

$1 \mathrm{mM} \mathrm{KCl}$ solution with the negatively charged fluorescent $0.2 \mu \mathrm{m}$ particle was filled into the microchannel by injecting the fluid with positive pressure from the reservoir. Initially, bubble was trapped in front of Nafion but pulling the pressure eliminated the bubble because PDMS is gas permeable material.

\section{Experimental setup}

An inverted fluorescence microscope (IX-51, Olympus, Japan) and a CCD camera (DP73, Olympus, Japan) were used to detect and trace the fluorescence-labeled samples. Commercial software (CellSense, Olympus, Japan) was used to synchronize the CCD camera with the microscope and to analyze the images. All captured images were analyzed by ImageJ.

\section{Measurement of the imbibition-induced flow velocity}

The flow velocity induced by imbibition through a nanoporous medium was measured by tracking the motion of the fluorescent microparticles near the reservoir of the microchannel since the diffusiophoretic force can be neglected near the reservoir. The images of the particle's motion were captured every $10 \mathrm{~s}$. Every $10 \mathrm{~s}$, the average velocities of particles were estimated as shown in Fig. 2.

\section{Results and discussions}

\section{The measurement of absorbing parameter $(S)$ of Nafion} nanoporous medium

Our previous work experimentally and theoretically presented that diffusiophoresis rate is always faster than imbibition rate when Nafion was used, since Nafion has

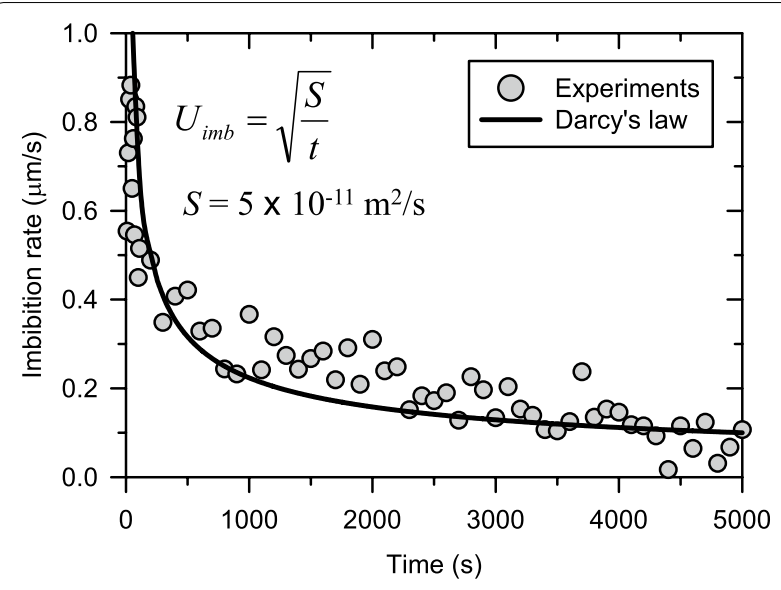

Fig. 2 The velocity graph of measured imbibition through Nafion (symbols) and regression curve derived from Darcy's law. $U_{\text {imb }}$ is the imbibition velocity, $t$ is the time, and $S$ is the absorbing parameter

high Donnan concentration. Also, the nanoporous medium's criteria for which diffusiophoresis or imbibition rate is faster were analyzed depending on the correlation between the permselectivity and the water-permeability of the medium [3]. Therefore, Nafion was chosen for investigating the effect of evaporation in this work. In order to characterize the absorption of Nafion, one need to obtain absorbing parameter, $S$ [3]. $S$ was calculated through the experiment of measuring particles' velocity induced by the imbibition of Nafion as shown in Fig. 2. The experimentations (circle symbols) can be fitted with Darcy's law [3] $\left(U_{i m b}=(S / t)^{0.5}\right)$ and $S$ was extracted as $5 \times 10^{-11} \mathrm{~m}^{2} / \mathrm{s}$. With given $L_{P D M S}$, one can derive $t_{1}$ (i.e. the time when the liquid reached to air through nanoporous medium) as $\left(L_{\mathrm{PDMS}}\right)^{2} /(4 S)$. Following section will describe the particle motion depending on $t_{1}$.

\section{The collapse of spontaneous particle exclusion depending on $t_{1}$}

The collapse time of spontaneous particle exclusion (or directional transition of particle) $\left(t_{2}\right)$ could be expected to be same with $t_{1}$. However, $t_{1}$ and $t_{2}$ was different in practice. For example, when $L_{\mathrm{PDMS}}$ was $250 \mu \mathrm{m}, t_{1}$ is $312.5 \mathrm{~s}$, while $t_{2}$ was measured to be around $60 \mathrm{~min}$ through the experiment as shown in Fig. 3a. This difference was explained by the schematic diagram shown in Fig. 3b. There were three distinguishable regimes of particle's motion as a function of time: (1) diffusiophoresis regime, (2) transition regime, and (3) evaporation regime. Under the nanoporous medium covered by PDMS, a particle was forced by dominant diffusiophoretic velocity in regime (1), where the spontaneous particle exclusion zone was generated. In the meantime, when the evaporation through nanoporous medium started because the 

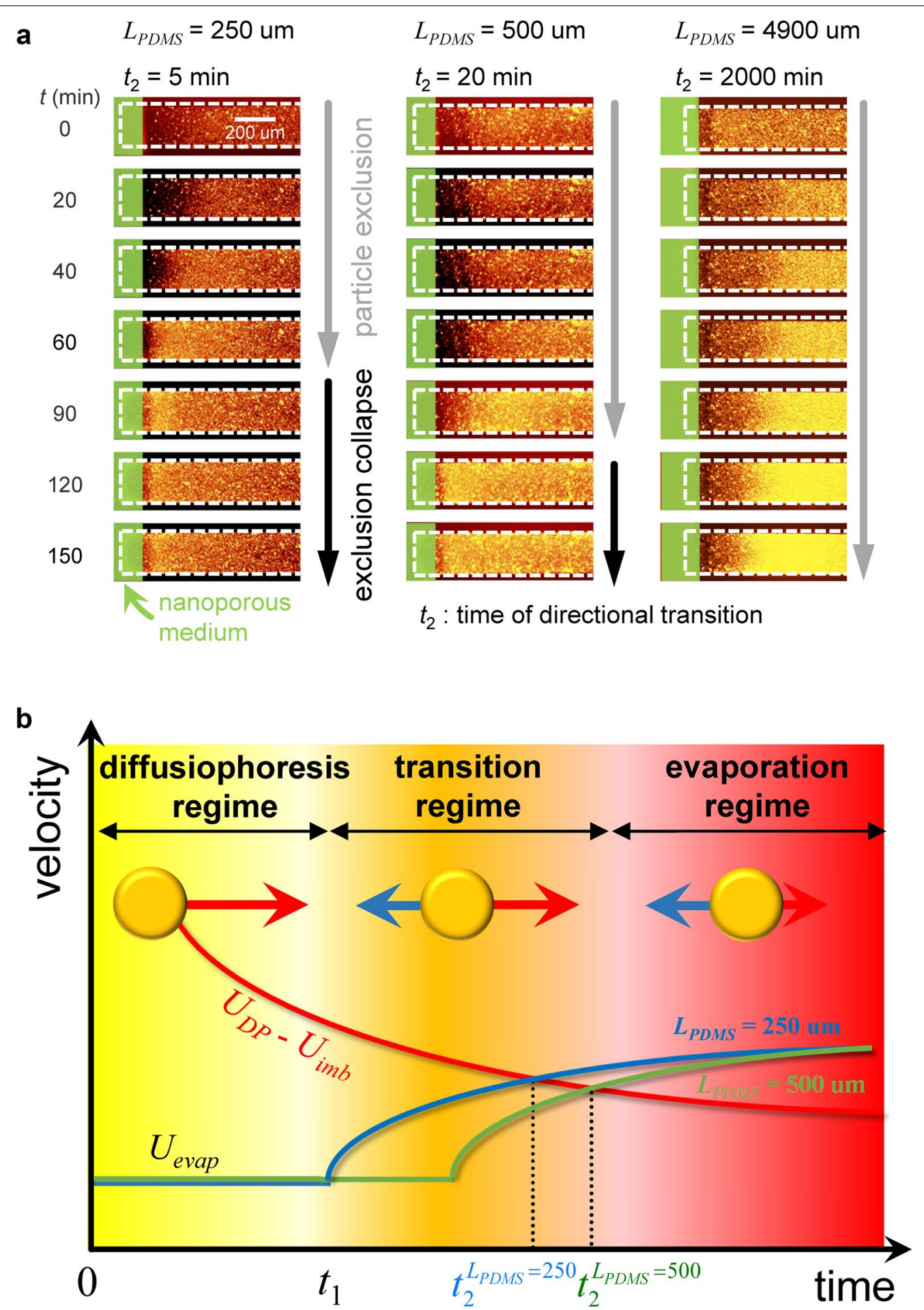

Fig. 3 a Time-evolving images of the particle's motion depending on $L_{\text {PDMs }}$. $\mathbf{b}$ The plot of three regimes about particle's motion affected by diffusiophoresis, imbibition, and evaporation. $t_{1}$ is the time when nanoporous medium was exposed to air (starting time of evaporation). $t_{2}$ is the collapse time of spontaneous particle exclusion zone. $0<t<t_{1}$ is diffusiophoresis dominant regime, $t_{1}<t<t_{2}$ is transition regime, and evaporation regime appears after $t_{2}$ 
medium was exposed to air, the particle was affected by additional velocity: $U_{\text {evap }}$ in regime (2). However, the particle's moving direction was not changed in this regime. Finally, when the evaporation velocity was faster than the sum of other velocities, particles eventually moved to opposite direction, which meant the collapse of the spontaneous particle exclusion zone. For example, there is no evaporation, but the particle moved with diffusiophoresis in $0<t<t_{1}$. In the range of $t_{1}<t<t_{2}$, the evaporation was started, but the direction of particle motion was not changed. Finally, the evaporation became to be dominant so that the direction of particle was reversed in $t>t_{2}$. At this moment, $U_{\text {imb }}$ still exists because the fluid loss by an evaporation at the end of Nafion should be refilled from an imbibition at the interface of Nafion/microchannel. Unless, Nafion should be completely dry so that the particle exclusion should appear again. However, we have not seen this, meaning that the imbibition and the evaporation simultaneously occur. This scenario was experimentally confirmed as shown in Fig. 3a. Furthermore, the effect of evaporation through nanoporous medium could be controlled by adjusting $L_{\mathrm{PDMS}}$. The collapse time $\left(t_{2}\right)$ of the shortest $L_{\mathrm{PDMS}}(250 \mu \mathrm{m})$ was faster than other length as shown in Fig. 3a. However, larger evaporation area has higher evaporation rate so that $t_{2}$ might be faster if one uses larger area.

The external convective flow due to evaporation through nanoporous medium as well as an imbibition would play a key role for characterizing the expansion of the spontaneous particle exclusion zone, since the velocity induced by evaporation affected particles' motion in the opposite direction to diffusiophoretic velocity.

\section{Conclusions}

In this work, the effect of evaporation through a nanoporous medium in addition to diffusiophoresis and imbibition is considered on the spontaneous particle exclusion zone. The experiment in which the time of starting evaporation effect was controlled by adjusting $L_{\text {PDMS }}$ showed that an evaporation played a deterministic role in characterizing the spontaneous particle exclusion zone. Therefore, this paper showed the possibility of adjusting particle's motion only by using external evaporation through nanoporous medium without any external power sources, and suggested a fundamental basis for designing spontaneous particle separation or preconcentration platform. Especially, evaporation has been neglected in the nanoscale fluidic phenomenon so that either one can utilize it for a separation application or one should prevent the evaporation to minimize undesirable errors. In addition, a separation by this evaporation effect could be suitable especially for separating electrosensitive biomolecules.

\section{Acknowledgements}

All authors acknowledged the supports from BK21 Plus program of the Creative Research Engineer Development IT, Seoul National University.

\section{Authors' contributions}

DL conducted the main experiment. JK fabricated devices. HL and SJK supervised the project. All authors wrote the manuscript. All authors read and approved the final manuscript.

\section{Funding}

This work is supported by the Basic Research Laboratory Project (NRF2018R1A4A1022513) and Mid-Career Project (NRF-2020R1A2C3006162) by the Ministry of Science and ICT.

\section{Availability of data and materials}

All data generated or analyzed during this study are included in this published article.

\section{Competing interests}

The authors declare no competing interests (both financial and non-financial).

\section{Author details}

${ }^{1}$ Department of Electrical and Computer Engineering, Seoul National University, Seoul 08826, Republic of Korea. ${ }^{2}$ Department of Chemical and Biological Engineering, Jeju National University, Jeju 63243, Republic of Korea. ${ }^{3}$ Inter-university Semiconductor Research Center, Seoul National University, Seoul 08826, South Korea. ${ }^{4}$ Nano Systems Institute, Seoul National University, Seoul 08826, South Korea.

Received: 9 March 2020 Accepted: 27 April 2020

Published online: 09 May 2020

References

1. Shin S, Ault JT, Warren PB, Stone HA (2017) Accumulation of colloidal particles in flow junctions induced by fluid flow and diffusiophoresis. Phys Rev X 7:041038

2. Ault JT, Warren PB, Shin S, Stone HA (2017) Diffusiophoresis in onedimensional solute gradients. Soft Matter 13:9015-9023

3. Lee JA, Lee D, Park S, Lee H, Kim SJ (2018) Non-negligible water-permeance through nanoporous ion exchange medium. Sci Rep 8:12842

4. Lee D, Lee JA, Lee H, Kim SJ (2019) Spontaneous selective preconcentration leveraged by ion exchange and imbibition through nanoporous medium. Sci Rep 9:2336

5. Lee H, Kim J, Yang J, Seo SW, Kim SJ (2018) Diffusiophoretic exclusion of colloidal particles for continuous water purification. Lab Chip 18:1713-1724

6. Choi J, Lee H, Kim SJ (2020) Hierarchical micro/nanoporous ion exchangeable sponge. Lab Chip 20:503-513

7. Park S, Jung Y, Son SY, Cho I, Cho Y, Lee H, Kim H-Y, Kim SJ (2016) Capillarity ion concentration polarization as spontaneous desalting mechanism. Nat Commun 7:11223

8. Duffy DC, McDonald JC, Schueller OJA, Whitesides GM (1998) Rapid prototyping of microfluidic systems in poly (dimethylsiloxane). Anal Chem 70:4974-4984

9. Son SY, Lee S, Lee H, Kim SJ (2016) Engineered nanofluidic preconcentration devices by ion concentration polarization. BioChip Journal 10:251-261

10. Kim SJ, Song Y-A, Han J (2010) Nanofluidic concentration devices for biomolecules utilizing ion concentration polarization: theory, fabrication, and applications. Chem Soc Rev 39:912-922

\section{Publisher's Note}

Springer Nature remains neutral with regard to jurisdictional claims in published maps and institutional affiliations. 